\title{
Transanal endoscopic surgery: patient selection and perspectives
}

\section{Andrea M Petrucci Dana R Sands}

Department of Colorectal Surgery, Cleveland Clinic Florida, Weston, FL, USA
Correspondence: Dana R Sands Department of Colorectal Surgery, Cleveland Clinic Florida, 2950 Cleveland Clinic Boulevard, Weston, FL 3333I, USA Tel +l 9546595000

Email sands@ccf.org
This article was published in the following Dove Press journal:

Open Access Surgery

22 August 2016

Number of times this article has been viewed

Abstract: Transanal endoscopic surgery is a 30-year innovation, which has allowed surgeons to treat patients with benign and early malignant rectal tumors using a minimally invasive approach. Its use in colorectal surgery continues to expand and be of interest for surgeons practicing in the minimally invasive surgical era. This review provides an overview of transanal endoscopic surgery, focusing on its different platforms, learning curve, and specific indications for benign and malignant diseases of the rectum. It reviews patient selection, with a focus on patient- and tumor-specific characteristics involved in the selection process, preparation for surgery, complications, and outcomes. Lastly, it reviews novel uses for transanal endoscopic surgery and future prospects.

Keywords: rectal tumor, rectal cancer, minimally invasive surgery, learning curve, benign disease, malignant disease

\section{Introduction}

Transanal endoscopic surgery (TES) was developed in the 1980s by professor Gerhard Buess with the aim of resecting lesions in the rectum that were not amenable to local excision or endoscopic resection. ${ }^{1}$ Transanal excision (TAE) is still commonly used today, mostly used for low rectal lesions located usually $7-8 \mathrm{~cm}$ from the anal verge. Patients with lesions not amenable to local excision are subjected to larger abdominal operations, which carry a much higher morbidity. The specialized equipment for TES allows surgeons to access lesions in the mid to upper rectum with improved exposure sparing patients from more complex procedures. The use of this minimally invasive technique has gained popularity and has become the standard approach for resecting primarily large adenomas and select carcinomas of the mid to upper rectum. In addition, experienced surgeons have explored the use of TES for repair of rectovaginal and rectourethral fistulas (RUFs), performing strictureplasty for anastomotic strictures of the rectum, repair of anastomotic leaks, resection of previous polypectomy sites with residual disease, excision of retrorectal tumors, and its potential use is continuously expanding. ${ }^{2}$ As with all new procedures, the surgical community was slow to adopt this approach due to its cost, its perceived difficulty as well as its limited use in colorectal surgery. ${ }^{3}$ Nonetheless, after many years of practice and robust clinical studies, TES has gained the interest of surgeons who prefer to treat their patients using more minimally invasive approaches.

The goal of this review is to provide a general overview of the TES approach with its different platform and applications, the utility of this approach, patient selection and preparation for surgery, complications, overall outcomes, and future directions. 


\section{Overview of TEM}

\section{Different platforms}

"Transanal endoscopic surgery" is a term used to describe a minimally invasive, incisionless approach for resecting lesions in the mid to upper rectum, extending up to the rectosigmoid junction. Since its description three decades ago, it has been a hot topic in the realm of colorectal surgery. It can be practiced using different platforms, all designed and constructed to help the surgeon with improved exposure and reach for challenging lesions in the rectum. Different types of TES equipment are available worldwide and can be classified as either a rigid or a soft platform, using similar instruments for dissection and suturing.

\section{Rigid platforms}

There are two units that are considered rigid. The first is the original transanal endoscopic microsurgery (TEM) platform (Richard Wolf, Knittlingen, Germany), used and described by Dr Buess, which consists of surgical instruments and an endosurgical unit. ${ }^{4}$ To gain access, a 4-cm diameter rectoscope, along with an introducer, is inserted into the rectum and sealed with an airtight faceplate through which a stereoscope with binocular vision and TEM-specific surgical instruments are inserted. The surgeon can decide whether to use the binocular vision or to project the operating field onto a monitor, allowing a wider audience to observe the procedure. The rectoscope is available in 12 - or 20 -cm lengths, with the option of using a flat or beveled tip to optimize exposure of the lesion. The entire unit is then secured onto the operating table using a multijointed clamp (Martin arm) and connected to the endosurgical unit, which provides constant $\mathrm{CO}_{2}$ insufflation to achieve pneumorectum, suction, and irrigation. ${ }^{5}$ The advantage of this operating platform is its ability to maintain pneumorectum using constant gas pressure in the rectum without insufflating the more proximal bowel, preventing unnecessary bowel distension and poor visualization. ${ }^{5}$ This very sophisticated and well-designed platform comes at a higher cost and therefore may be a limiting factor for adopting this technique for surgeons who are starting to practice TES. Nevertheless, it has been proven to be cost-effective when compared to open resection in high volume centers, mainly driven by the shorter hospital stay. ${ }^{5-7}$

The second rigid platform is the transanal endoscopic operation (TEO; Karl Storz, Tuttlingen, Germany). This less-expensive alternative allows surgeons to use standard laparoscopic instruments, including a $5 \mathrm{~mm} 30^{\circ}$ laparoscope and overall laparoscopy setup, making this platform more affordable, and may be more attractive for surgeons with prior laparoscopic experience. ${ }^{8}$ As with the TEM platform, the beveled rectoscope is $4 \mathrm{~cm}$ in diameter, but this option comes in three different sizes: $7.5,15$, and $20 \mathrm{~cm}$. Instruments ranging from 3 to $14 \mathrm{~mm}$ can be inserted into the channels of the rectoscope, including staplers and ultrasonic devices. The rectoscope is secured to the operating table using a holding system and articulated stand. ${ }^{9}$ TEO differs from TEM in that it is not equipped with binocular vision or a dedicated suction insufflation device. ${ }^{8}$

\section{Soft platform}

Transanal minimally invasive surgery (TAMIS) is the most recent and most affordable platform for TES. Originally described by Atallah et $\mathrm{al}^{10}$ in 2010 as a hybrid between TEM and single-port laparoscopy, this platform uses a lubricated single-incision laparoscopic surgery port (SILS Port; Covidien, Mansfield, MA, USA) to gain access to the rectum, with its upper border anchoring at the anorectal ring. It has a 3 $\mathrm{cm}$ diameter neck and three $5 \mathrm{~mm}$ cannulas for single-port surgery along with a separate access used for insufflation. Standard ordinary laparoscopic instruments are used to perform the procedure with the use of more specialized articulating instruments where applicable. Another device approved by the Food and Drug Administration is the GelPOINT Path Transanal Access Platform (Applied Medical, Rancho Santa Margarita, CA, USA), specifically designed for TAMIS procedures. ${ }^{8}$ At the time of introduction of TAMIS, it was found to be a feasible, less expensive alternative to TEM with short operating times and no morbidity or mortality at short-term follow-up. ${ }^{10}$ Different ports for TAMIS have been developed throughout the years, with port lengths (referring to the actual working port length, not accounting for the external part of the device) ranging from 37 to $44 \mathrm{~mm}$ and port diameters between 35 and $40 \mathrm{~mm}$, depending on the brand. ${ }^{11}$

\section{Learning curve}

Similar to the early days of laparoscopy, TES was not well accepted decades ago and was viewed as difficult, expensive, and with limited indications. ${ }^{3}$ Since then, a multitude of studies have been published showing the benefits of TES, encouraging surgeons to adopt this new wave in colorectal surgery. Courses and workshops around the globe have allowed surgeons with different levels of experience and expertise to train on the different TES platforms, teaching the basics of setup and surgical technique. As with any new procedure, there is a learning curve that surgeons must embark on before achieving a certain proficiency level. This learning curve may differ among surgeons, given that every 
individual has a different basic skill set and experience with minimally invasive surgery. Noticeably, surgeons trained in the prelaparoscopy era may likely require more coaching and practice as opposed to those who regularly practice laparoscopy. Among the studies assessing the learning curve of surgeons adopting this technique, one retrospective study assessed the learning curve effect on conversion, procedure length, complications, and recurrence rate of four surgeons who performed a total of 693 rectal lesion excisions. They demonstrated that the learning curve affected the first three outcomes but did not affect the recurrence rate in patients after a 16-month follow-up, which is likely explained by evolving patient populations. ${ }^{12}$ Although a learning curve effect was calculated in this study, there was no mention of an actual number of cases required before a surgeon demonstrates a certain level of proficiency with this procedure. A recent retrospective study by Maya et $\mathrm{al}^{3}$ assessed the learning curve of a single surgeon performing TEM using the Wolf platform in 23 cases. Using a Cumulative sum technique, which is an objective assessment tool of individual surgeon performance, they were able to demonstrate stabilization of the learning curve for TEM after four cases. In addition, the operative time as well as the average rate of rectal lesion excision using TEM significantly decreased after four cases (mean operating time of 130.5 minutes for specimens with a mean size of $16.6 \mathrm{~cm}^{2}$ with minimal fragmentation and morbidity). ${ }^{3}$ This is encouraging for surgeons who are interested in learning this technique given that the learning curve may not be as steep as initially anticipated on the basis of the results of this study. However, it is important to realize that with any new technique, steady practice is important to maintain skills and improve performance over time.

\section{Utility of TES}

TES has become the standard approach for resection of rectal adenomas and early rectal cancers with favorable histology. However, its use has expanded far beyond its original description by Professor Buess 30 years ago for resection of rectal tumors. Its potential continues to be challenged as surgeons are pushing the limits of transanal surgery using TES for repair and resection of other colorectal problems. Without a doubt, this minimally invasive technique spares the patient from invasive abdominal surgery, which would be the gold standard procedure for early lesions of the mid and upper rectum not amenable to TAE. The development of TES techniques, regardless of the platform used, provides surgeons with specialized equipment that improves illumination and exposure of the surgical field with better reach for lesions higher up in the rectum to allow for a more precise dissection and less morbidity. The advantage of TES is that virtually any rectal adenoma can be excised regardless of the size, extent of circumferential involvement, and height (provided that it is below the rectosigmoid junction). ${ }^{13}$

\section{TES compared to other local procedures}

With the advent ofTES, there has been a paradigm shift in the treatment of rectal adenomas and very early rectal cancers. Endoscopic approaches such as endoscopic mucosal resection (EMR) or endoscopic submucosal dissection (ESD), which are often used to resect lesions in the colon or upper gastrointestinal tract, can be quite challenging and may not be the procedure of choice for patients with rectal lesions. Moreover, EMR would not be an acceptable approach for patients with early rectal cancers given that $\mathrm{T} 1$ cancers invade the submucosa. A study by Barendse et $\mathrm{al}^{14}$ comparing EMR to TEM for large adenomas $(>2 \mathrm{~cm})$ found EMR to be less effective at removing the lesion on the first attempt. However, EMR appeared to be as successful as TEM at removing the remnant lesion on the second attempt. Moreover, patients who had undergone EMR had a higher early recurrence rate at 6 months follow-up endoscopy (30\% vs $10.2 \%$ for EMR and TEM, respectively). ${ }^{14}$ Furthermore, piecemeal resection of a lesion makes it more difficult for adequate histopathological assessment and has been found to have higher recurrence rates at the polypectomy site. ${ }^{15,16} \mathrm{ESD}$ offers a more complete en-bloc excision of larger, sessile polyps, typically $>20 \mathrm{~mm}$ with a lower recurrence rate compared to EMR. ${ }^{17}$ Although it is commonly used in the upper gastrointestinal tract, its use in the colon and rectum is more rare. TEM is technically challenging with a steep learning curve making it difficult for surgeons to adopt this technique over conventional TAE. One systematic review and meta-analysis comparing ESD to TEM in $>2,000$ patients with noninvasive rectal lesions found patients undergoing TEM had a higher rate of $\mathrm{R} 0$ resection due to the ability to perform a full-thickness excision compared to patients undergoing ESD. Moreover, patients post-ESD were more likely to undergo further abdominal surgery due to complications or pathological indications compared to TEM. Both the approaches had similar complication profiles. ${ }^{18}$

Conventional TAE initially described by Parks for rectal lesions has been practiced for years and has been the go-to operation for distal rectal adenomas not amenable to endoscopic resection as well as early rectal cancers. Patients who qualify for a full-thickness TAE for carcinoma have lesions that are well-to-moderately differentiated superficial rectal cancers (T1), 
$<4 \mathrm{~cm}$ in size, located $<10 \mathrm{~cm}$ from the anal verge, with $<40 \%$ circumferential involvement, without any perineural or lymphovascular invasion. ${ }^{19}$ This operation continues to be widely practiced because it offers patients an oncologic resection while sparing them the morbidity of an abdominal operation. Although technically challenging due to the narrow working space, difficult reach, and poor visualization in the proximal rectum, patients who undergo TAE are spared the need for more radical abdominal surgery, which leads to decreased postoperative morbidity, improved functional outcomes and avoidance of possible stoma creation due to its less invasive approach. ${ }^{2,20}$ In 2008, Moore et $\mathrm{al}^{21}$ published the first large study comparing the effectiveness of TAE to TEM. Although this was a retrospective review, it looked at 171 patients who underwent local excision for both adenoma and carcinoma ( $n=89$ forTAE; $n=82$ for TEM). For both groups, there was a significantly higher rate of negative margins after resection as well as less specimen fragmentation in the TEM group. The recurrence rate for adenomas was significantly lower in the TEM versus the TAE group ( $3 \%$ vs $32 \%$, respectively); for carcinomas, there was a strong trend toward decreased recurrence in the TEM group, although this was not statistically significant. ${ }^{21}$ These findings were reproduced in other studies. De Graaf et al ${ }^{22}$ compared TAE to TEM for adenomas and had similar findings with regard to fragmentation and resection margins. Interestingly, patients with positive margins in the TEM group had lower recurrence rates than did patients with positive margins in the TAE group (7\% vs 59.6\%, respectively), concluding that TEM is superior to TAE for rectal adenomas. ${ }^{22} \mathrm{~A}$ recently published systematic review and meta-analysis by Clancy et $\mathrm{a}^{23}$ compared recurrence rates and pathologic outcomes between TEM and TAE. The authors demonstrated that patients undergoing TEM had a higher rate of negative margins, less specimen fragmentation, and lower lesion recurrence rates with similar complication profiles for both the procedures, but without any significant differences between the groups. However, the studies included were mostly retrospective. ${ }^{23}$ With these findings and the current available technology for TES, it is difficult to justify using TAE alone without the addition of TES, either as a combined procedure or alone, to treat patients with rectal neoplasia. Regardless of the platform applied, TES allows surgeons the opportunity to improve visualization with a more functional operating system to optimize exposure and enhance surgical resection.

\section{TES and rectal adenomas}

The use of TES is quickly gaining popularity within the field of colorectal surgery. Much of the ongoing research is currently exploring different uses for TES. However, the most widely accepted and least debated indication includes resection of any rectal adenoma below the rectosigmoid junction, regardless of its size or circumferential involvement. Rectal adenomas are direct precursors of rectal cancer; therefore, complete excision is necessary to avoid the development of dysplasia and, subsequently, carcinoma. It is also well known that adenomas $>1 \mathrm{~cm}$ in size carry a $38.5 \%$ chance of harboring high-grade dysplasia (HGD) or carcinoma. For this reason, complete resection of these adenomatous lesions reduces the long-term risk of colorectal cancer. ${ }^{24,25}$ Although TAE is a procedure commonly performed for distal rectal lesions, it is not ideal for lesions higher up in the rectum. Other approaches such as the posterior trans-sphincteric (York-Mason), trans-sacral (Kraske), or an abdominal low anterior resection are invasive procedures for patients diagnosed with a benign disease that carry a significantly higher overall morbidity and can lead to poor functional outcomes. ${ }^{26}$ Its use for larger adenomas has been extensively studied and proven to be more effective than other local resection methods, such as EMR and ESD, with higher rates of negative resection margins and less piecemeal specimens, resulting in a lower recurrence rate. ${ }^{27}$ For these benign precancerous lesions, regardless the platform used, TAMIS/TEM/TEO, it is imperative to fully resect the lesion with negative margins to decrease the recurrence rate, as the latter is dependent on margin status.

\section{TES and rectal cancer}

According to the American Cancer Society, colorectal cancer is the third most common cancer diagnosed in the USA, with an estimated incidence of 39,220 new cases of rectal cancer for 2016. The estimated 5-year survival for patients with stage 1 rectal cancer is $87 \%$, which comprises patients with pathological stages T1 and T2 with node-negative disease. Treating these patients can be challenging, and surgery plays an important role. The standard of care for curative treatment is a total mesorectal excision (TME), either with a low-anterior resection or an abdominoperineal resection, depending on the height of the tumor, to ensure complete resection of the lesion along with its mesorectal lymph nodes. For patients with early stage rectal cancer, this can potentially be curative; however, this operation carries a significant morbidity $(30 \%-68 \%)$ and mortality $(0 \%-6.5 \%) .{ }^{28}$ In a select group of patients with early rectal cancer, TES can be an option to decrease postoperative morbidity and improve quality of life as long as their oncologic outcome is not compromised. Nonetheless, not all early rectal cancers are amenable to local excision as studies have shown that patients with T1 tumors 
who undergo TAE have recurrence rates reportedly between $0 \%$ and $31 \%$, which questions which factors come into play to allow for such discrepancies in recurrence rates in patients who are staged with node-negative disease..$^{29}$ These factors include T-stage along with certain histopathological markers that have been shown to have a higher risk of lymph node metastasis and hence recurrence.

The most important predictor of lymph node metastasis is the T-stage of the tumor as the risk of nodal metastasis increases with increasing T-stage: $0 \%-12 \%$ risk of nodal positivity for T1 tumors, $12 \%-38 \%$ for T2 tumors and is significantly higher for $\mathrm{T} 3$ and $\mathrm{T} 4$ tumors with risks ranging from $36 \%$ to $79 \%$. $^{30,31}$ Patients with tumors staged as T2 and above should not be considered for TES as their risk of lymph node metastasis is too high, and these patients should undergo formal TME even without any gross nodal involvement on staging workup. A study by Elmessiry et al $^{28}$ comparing local excision and TME in T1 and T2 cancers found a higher local recurrence $(P=0.025)$ and lower disease free survival $(P=0.044)$ for T2 lesions after local excision, suggesting that these tumors are best treated with TME. Moreover, for patients who have recurrence after local excision for early rectal cancer and recur, the outcome of salvage surgery is also affected by the initial operation. Mellgren et $\mathrm{al}^{30}$ looked at local excision versus radical surgery in patients with T1N0 and T2N0 disease and found that in patients with locoregional recurrence after local excision, salvage surgery was more effective in $\mathrm{T} 1$ cancers and that local excision for T2 cancers may compromise their overall survival. Tumor size is also thought to be a relative contraindication $(>3-4 \mathrm{~cm})$ to TES due to difficulties with exposure, hence a less effective resection with inadequate margins which may contribute to higher locoregional recurrence. ${ }^{2}$

T-stage and size are not the only factors taken into account when evaluating a patient for possible TES. The histopathology of the tumor has significant implications on the decision making for possible TES since certain features are more ominous and would favor radical excision over TES. First, the degree of submucosal involvement is an important predictor of lymph node metastasis. The Kikuchi classification divides the submucosa into three depths of involvement: sm1 describes slight submucosal invasion, sm2 is intermediate invasion, and sm3 is deep invasion abutting the inner surface of the muscularis propria. Kikuchi et $\mathrm{al}^{32}$ demonstrated that sm 3 tumors have the highest risk of lymph node metastasis and advised to treat these tumors with formal bowel resection. Moreover, it is also important to distinguish the morphology of the polyp as submucosal involvement in pedunculated and sessile polyps is treated differently. Pedunculated lesions that harbor cancer can be assessed using Haggitt's classification: 1 through 4, where Haggitt levels 1-3 describe a lesion where carcinoma invades the submucosa in the head, neck, and stalk, respectively, and can be resected with simple polypectomy granted that the resection margins are clear. Haggitt level 4 signifies invasion into the base of the lesion, just above the muscularis propria. Haggitt's level 4 is a higher risk lesion and can be classified as either sm2 or sm 3 and therefore should be treated with caution when assessing a patient for TES.

In addition to submucosal invasion, lesions need to be assessed for their degree of differentiation, where poorly differentiated lesions are higher risk than well-to-moderately differentiated tumors. The presence of perineural or vascular invasion, any mucinous component, and, more recently, tumor budding at the margin are also ominous histopathological features of higher risk lesions. Blumberg et $\mathrm{al}^{33}$ looked at pathological factors and the risk of lymph metastasis in rectal cancer and found node differentiation and lymphatic vessel invasion to affect the risk of lymph node metastasis. Both welldifferentiated and moderately differentiated tumors had a 14\% risk of lymph node metastasis, whereas the risk increased to $30 \%$ for poorly differentiated lesions. Similarly, lesions with lymphovascular invasion had a 33\% chance of lymph node metastasis, whereas those without lymphovascular invasion only had a $14 \%$ risk of positive nodes. ${ }^{33}$ Nascimbeni et $\mathrm{al}^{34}$ studied T1 rectal lesions of the rectum and found that lymphovascular invasion, sm3 depth of invasion, and location in the lower third of the rectum to be associated with a higher rate of lymph node metastasis. With this in mind, careful histopathological assessment of tumors is crucial and careful selection is necessary when considering a patient for TES.

In summary, patients who should be considered for TES for early rectal cancer are those with well- to moderately differentiated T1 tumors, with superficial invasion of the rectal wall into the submucosa ( $\mathrm{sm} 1$ ) excluding the muscularis propria, without any signs of perineural or lymphovascular invasion, or any tumor budding or any mucinous component. ${ }^{2}$ Within this group of patients, it is reasonable to offer them TES with appropriate postoperative surveillance as studies have shown similar oncologic outcomes with radical surgery for carefully selected low-risk T1 tumors. ${ }^{35,36}$ In a very small subgroup of patients with higher risk lesions, TES alone or combined with adjuvant treatment and close surveillance may be offered. These include patients with advance disease who may benefit from a local procedure if the lesion is amenable to resection. Also, patients who refuse to have radical surgery 
due to fear of surgical outcome or fear of stoma and who are reliable for close surveillance may undergo TES followed by chemoradiation if they agree to the risks and understand the poorer oncologic outcome of this approach. ${ }^{2}$

\section{Other uses for TES}

TES is most often used for benign and malignant rectal neoplasia; however, many surgeons have attempted to use TES to treat other colorectal diseases, including but not limited to rectovaginal fistula (RVF), RUF, stricture, and anastomotic leak. A review by D'Ambrosio et $\mathrm{al}^{37}$ looked at the use of TES for the repair of RVF using the rigid TEM platform in 13 reported cases. Using a combination of TES and manual dissection, a layered closure was performed thus sparing patients from a perineal incision. The main drawback described was the lack of rectal visualization during dissection of the rectovaginal septum, which had to be performed manually by the surgeon. Despite this, results have shown an overall healing rate of $93 \%$, with minor complications in $15 \% .{ }^{37}$ In addition, Darwood and Borley ${ }^{38}$ described using TES for high RVF using a mucosal advancement flap, stating excellent visualization and low morbidity. ${ }^{38}$ A similar approach has been used for repair of RUF. Reports as far back as 1996 have described RUF repair using a combination of cystoscopy and full-thickness rectal dissection with layered closure using TES, advocating for meticulous dissection and debridement of the fistula for successful closure. ${ }^{39}$ In a subsequent report, Bochove-Overgaauw et $\mathrm{al}^{40}$ used TEM in two patients who developed RUF after prostate surgery and reported one failure attributed to extensive scar tissue following previous gracilis flap repair.

Another use for TES described in the literature is for the treatment of anastomotic strictures. A case report by Kato et $\mathrm{al}^{41}$ described the use of TEM with a Nd:YAG laser for the treatment of an anastomotic stricture by fulgurating the rectal wall with no associated complications. ${ }^{41} \mathrm{~A}$ more recent publication by Baatrup et $\mathrm{al}^{42}$ described TEM for stricture using a full-thickness hemicircumferential incision from 4 to 8 o'clock and closure of the defect, in six patients. Aside from technical issues encountered in one patient with a very low stricture, this approach was feasible, well-tolerated, and with no complications after 16 months follow-up. It was, however, technically challenging. ${ }^{42}$

Other applications for TES have been described, including excision of rare tumors, including carcinoids, gastrointestinal stromal cell tumors, and retro-rectal tumors. Carcinoid tumors are most common in the appendix; however, they also occur in the rectum and can be locally excised if $<1 \mathrm{~cm}$ in size. They are mostly asymptomatic and lesions $<1 \mathrm{~cm}$ are rarely metastatic ${ }^{43}$; therefore, local excision is appropriate. Kinoshita et $\mathrm{al}^{44}$ studied 27 patients who underwent TEM for carcinoid tumors, with minor complications occurring in two patients, including temporary soilage and dehiscence. ${ }^{44}$

Other reported innovative uses include schwannoma, removal of a foreign body and even rectal prolapse, using a technique that resembles the stapled transanal rectal resection procedure. . $^{4,46}$

\section{Patient selection and preparation Patient preparation for surgery}

First, a detailed history focusing on the patient's medical comorbidities, current medications, allergies, previous colonoscopies or any pertinent intervention (such as biopsies), or surgery (especially in the anorectal area) the patient may have undergone is essential for future surgical planning. This also helps the surgeon assess possible risks of anesthesia and any potential problems encountered in the perioperative period. Moreover, documenting any current issues with continence or change in bowel habits is important to understand preoperatively so that any changes in the postoperative period can be monitored and investigated, if necessary. Next, the physical examination allows the surgeon to evaluate the patient as well as the lesion in question. Patients with proven adenomas or a select few patients with carcinoma of the rectum, which are not resectable using endoscopic techniques, can be evaluated for TES. Every patient who is considered for TES must first undergo digital rectal examination to assess location (anterior vs posterior or right vs left lateral), height (whether the lesion is palpable by digital rectal examination or not), and palpation (soft vs. firm) of the lesion. These diagnostic parameters will allow the surgeon to begin the planning process for eventual excision. Following digital rectal examination, patients must undergo a flexible or rigid sigmoidoscopy to characterize the lesion according to its size, distance from the dentate line, circumferential involvement, and relationship to the rectal valves or any other structure that may have adhered to the lesion. This is imperative for preoperative planning. Once completed, the patient must undergo a full colonoscopy to assess for possible synchronous polyps or neoplasia, if not recently performed.

Patients with suspicious appearing lesions on physical examination with or without pathologically confirmed adenomas may have to undergo further imaging to better characterize the lesion in case of underlying malignancy prior to any local excision. Imaging includes endorectal ultrasound (ERUS) and pelvic magnetic resonance imaging (MRI). 
Both the modalities have been studied extensively regarding their accuracy in detecting wall invasion (T-stage) and lymph node involvement for rectal cancer. One study found MRI to be slightly superior in detecting wall invasion when compared to ERUS (89.7\% vs $85.29 \%$ ) with a similar value of detecting lymph node involvement (74.5\% vs $76.47 \%) .{ }^{47}$ A meta-analysis comparing ERUS, computed tomography scan, and MRI found ERUS to be more accurate at assessing local invasion, whereas all the three modalities were found to have similar sensitivities for detecting lymph node involvement. ${ }^{48}$ Computed tomography and MRI have also been used to evaluate iliac, mesenteric, and retroperitoneal lymph nodes, as opposed to ERUS that specifically assesses mesorectal lymph nodes. In addition, MRI is helpful for higher rectal lesions. ${ }^{8}$ Although these imaging modalities are acceptable, a greater trend is growing toward using MRI, since it is more widely available than ERUS; the latter is also operator-dependent and may not be available in all centers.

Prior to surgery, patients are instructed to take a full mechanical bowel preparation or two rectal enemas. The advantage of a full bowel preparation is to avoid any contamination of the surgical field from proximal, nonevacuated feces, which may obstruct the view. On the day of surgery, patients receive preoperative prophylactic antibiotics, and the procedure is generally performed under general anesthesia. In certain circumstances, TES can be undertaken under regional spinal anesthesia. A Foley catheter is inserted to decompress the bladder, and the patient is positioned so that the lesion is located at 6 o'clock. For TAMIS, the procedure can be undertaken in the lithotomy position, regardless of the location of the lesion; however, for anterior lesions, patients can be placed in the prone position. ${ }^{10}$ Postoperative management is surgeon- or center-dependent. Some patients are discharged home the same day of surgery, while others are admitted to hospital. In general, patients receive a dose of postoperative antibiotics, although duration of treatment is not standardized, and there is no evidence to date justifying prolonged use of antibiotics. Patients with lesions requiring further surgical intervention after full-thickness excision (such as lesions with positive margins) should do so in 4-6 weeks to allow the bowel time to heal. Patient with more advanced malignancies diagnosed after local resection should undergo radical surgery for optimal oncologic management.

According to the NCCN guidelines, surveillance for completely resected adenomas consists of a repeat colonoscopy in 1 year. For patients with early rectal cancer with complete resection and no worrisome features warranting further intervention, patients should be followed up with a history and physical examination, carcinoembryonic antigen levels, chest, abdominal and pelvic computed tomography, and proctoscopy every 3-6 months for the first 2 years, then every 6 months thereafter for a total of 5 years. A colonoscopy should be performed after 1 year. ${ }^{49}$

\section{Procedure-associated complications}

In general, TES is safe with a minimal complication profile. For TAMIS and TEM, overall complication rates have been reported as $7.5 \%$ and $10.5 \%$, respectively, and generally include peritoneal entry, vaginal or urethral injury, sphincterrelated injury leading to incontinence, bleeding, and wound dehiscence. ${ }^{11,50}$ Most of the complications that can occur intraoperatively are well managed using the TES equipment.

For TEM, peritoneal entry should not be considered a dreaded problem. This usually occurs for lesions located in the anterior upper rectum. Gavagan et $\mathrm{a}^{51}$ studied 34 patients who underwent TEM for benign and rectal lesions, with entry into the peritoneum in eleven patients, all repaired using TEM, and 23 patients without entry into the peritoneum. They found no major complications in patients with peritoneal entry without any differences in perioperative complications. In addition, peritoneal entry does not mandate conversion to laparotomy. ${ }^{51}$ The available visualization and instrumentation make it possible for the surgeon to close the defect immediately when it occurs. If needed, there is always the possibility of performing a laparoscopic-assisted closure and, rarely, a laparotomy. There are less published studies available for the TAMIS, making it more difficult to assess its effectiveness for peritoneal entry. A recent study by Molina et $\mathrm{al}^{52}$ assessed peritoneal entry for all the three TES platforms (TAMIS, TEO, and TEM) and concluded that addressing peritoneal entry after resection of upper rectal lesions was more difficult with TAMIS, requiring conversion to a rigid platform or assistance using an abdominal approach more often than the other two platforms.

Guerrieri et $\mathrm{al}^{53}$ evaluated complications in 588 patients who underwent TEM for rectal adenoma and found that, in their cohort, $8.1 \%$ of patients experienced minor complications (such as suture site leakage, soiling, and minor postoperative bleeding) while only $1.2 \%$ experienced major complications (such as rectal hemorrhage requiring intervention, RVF requiring ileostomy, rectovesical fistula, suture site leakage treated with second TEM). In a recent systematic review of TAMIS looking at 390 procedures performed worldwide, there was a total of 29 complications, where five were classified as Clavien-Dindo grade 3, which included peritoneal entry and bleeding requiring an intervention. The 
remaining patients suffered grade 1 and 2 complications, with the most common one being self-limiting bleeding in ten patients. ${ }^{11}$

Although wound dehiscence is likely a more common complication, the wound is not routinely evaluated and examined in the immediate postoperative period. ${ }^{2}$ Regardless, when diagnosed, treatment consists of antibiotics and local therapy depending on the patient's symptoms. ${ }^{5}$ In the event of a suture dehiscence causing a phlegmon or abscess, treatment may require colostomy creation in order to allow the area to heal. In a retrospective study by Guerrieri et al, ${ }^{54}$ evaluating their experience with TEM for resection of rectal adenoma, only one of their 402 patients experienced a large retroperitoneal phlegmon requiring radiologic drainage and diverting colostomy. ${ }^{54}$ Postoperative hemorrhage has also been reported, ranging anywhere between $1 \%$ and $13 \%$, usually managed conservatively, and rarely requiring transfusions. ${ }^{5}$ Stricture formation has also been reported. Flexer et $\mathrm{al}^{55}$ looked at their experience using the TEM platform and reported stricture formation in six out of 164 patients treated successfully with endoscopic or intraoperative dilatation. ${ }^{55}$

\section{Outcomes}

\section{Functional}

Functional issues may severely impact a patient's quality of life. Regardless of the platform used, there is always a concern that operating through the anus with continuous insufflation and distension of the rectum may cause damage to the sphincters, which may lead to fecal soiling and incontinence. Fortunately, studies considering patients' anorectal function after undergoing TES have shown encouraging results. Specifically, there are several studies that have looked at functional outcomes after TEM, given that it entails a large, rigid rectoscope inserted through the anus for a prolonged period of time. In a small prospective study, Herman et $\mathrm{al}^{56}$ assessed the risk factors for anorectal dysfunction after TES and found that $\sim 50 \%$ of their patients had disturbances in anorectal function at 3 weeks, with partial-to-moderate dysfunction in $21 \%$ at 6 months after TES. The most common risk factors were preoperative disturbances in anorectal function, pre- or postoperative abnormalities on endoanal ultrasound, and the extent of excision of the lesion (lesions that were $>50 \%$ of the circumference and full-thickness excisions). Nevertheless, functional issues were temporary and appeared worse in patients with the aforementioned risk factors. ${ }^{56}$. Jin et $\mathrm{al}^{57}$ assessed 37 patients who underwent TEM for rectal tumors, including adenomas and carcinomas. All patients had normal preoperative anorectal manometry, and any patient who had undergone previous anorectal surgery was excluded. Over a 6- to 20-month follow-up period, Jin et $\mathrm{al}^{57}$ found that low anorectal pressures, postoperative internal anal sphincter defects, and depth of tumor excision from TEM all contributed to incontinence during the early postoperative period; however, incontinence was transient and anorectal function eventually returned to baseline in all the patients. ${ }^{57}$ Zhang et $\mathrm{l}^{58}$ looked at anorectal function after repeated TEMs from presumed repeated injury to the internal anal sphincter in a group of patients who had undergone a subtotal colectomy with ileorectal anastomosis for familial adenomatous polyposis. Patients underwent repeated TEM for excision of rectal polyps in the remaining rectum. The authors found that, although anorectal parameters studied on endoscopic ultrasound changed over time, patients' function was well-preserved with a good quality of life. ${ }^{58} \mathrm{~A}$ larger study by Allaix et $\mathrm{al}^{59}$ assessed long-term outcomes, including quality of life, and sexual, urinary, and sphincter function, in patients with rectal cancer undergoing TEM. In their cohort of 93 patients, there was an increase in incontinence and manometry scores by 3 months after surgery with return to their baseline at 60 months follow-up. Quality of life improved by 12 months follow-up, and there were no problems with urinary or sexual function associated with TEM. ${ }^{59}$

Studies looking at TAMIS and postoperative anorectal function have shown similar results, despite the use of different equipment. A recent study ${ }^{60}$ in a small cohort of patients undergoing TAMIS for benign and malignant tumors found that nine patients had normal incontinence scores postoperatively, with only one patient having abnormal scores that resolved at 6 weeks after surgery. The only abnormality on anorectal manometry was a lower mean minimum rectal sensory volume at 3 weeks after surgery. ${ }^{60}$ Another study by Verseveld et $\mathrm{al}^{61}$ in 24 patients undergoing TAMIS found no deleterious effect of TAMIS on anorectal function; only five patients had increased fecal incontinence scores, and these five patients were found to have more distal tumors that were significantly larger in size. ${ }^{61}$ Lee et al $^{62}$ studied the outcomes in patients undergoing TAMIS under spinal anesthesia and found no abnormalities on endoanal ultrasound 3 months after surgery. ${ }^{62}$ The platform used in TAMIS is softer than the rigid platforms, with a smaller diameter therefore is thought to lead to less sphincter injury. ${ }^{62}$

\section{Recurrence}

As discussed earlier, TES has become the modality of choice for resecting rectal tumors, and one advantage has been the decrease in recurrence rates. With regard to recurrences in adenomas, resection margin is one of many factors 
contributing to local recurrence. The recommended margin of clearance for adenomas is $5 \mathrm{~mm}$ circumferentially, marked with coagulation dots that guide the surgeon around the lesion. ${ }^{63}$ An early review by Smith et $\mathrm{al}^{64}$ of 150 patients undergoing TEM, 82 for resection of adenomas, reported a recurrence rate of $11 \%$. All adenomas that recurred were small and easily treatable. ${ }^{64}$ Since then, may studies have looked at the adenoma recurrence rate. A retrospective study by Allaix et $\mathrm{al}^{27}$ found the resection margin to be the only independent predictor of recurrence on multivariate analysis after a 12-month follow-up. In this same study, the size of the lesion was not associated with recurrence on multivariate analysis; however, this did reach statistical significance on univariate analysis, with lesions $>5 \mathrm{~cm}$ having a higher recurrence risk. Conversely, dysplasia type showed a trend toward higher recurrence with HGD, but again this was not statistically significant. ${ }^{27}$ However, a retrospective study by Ganai et $\mathrm{al}^{65}$ did find that size and presence of HGD were risk factors for recurrence in a retrospective analysis of 107 patients, where $45 \%$ of patients had adenomas and $17 \%$ had adenoma with HGD. ${ }^{65}$ In a study by Said and Stippel, ${ }^{66}$ looking at their experience with TEM for sessile adenomas over a 10 -year period, they found the 1- and 5-year recurrence rate to be $1.2 \%$ and $7 \%$, respectively, with a relationship with size and histological type of the adenoma. ${ }^{66}$

When looking at recurrence for $\mathrm{T} 1$ rectal cancers, it is important to distinguish "low-risk" from "high-risk" cancer, as it has been shown that, with the latter, recurrence rates are higher when tumors contain the histopathological features that predispose them to a higher risk of recurrence. Borschitz et $\mathrm{a}^{67}$ assessed the influence of histopathological criteria on recurrence in patients with $\mathrm{T} 1$ rectal cancer. They divided the groups into "high-risk" and "low-risk," based on histological criteria, tumor localization, size, and degree of resection. "High-risk" tumors included those that exhibited high-risk histopathologic markers, had R1 resection margins, and were a result of tumor fragmentation, whereas "low-risk" tumors had an R0 resection. They found that the "low-risk" tumors had a recurrence rate of $6 \%$, compared to $39 \%$ in the "highrisk" group. Moreover, patients in the "high-risk" group who had undergone reresection for recurrence decreased the local recurrence rates to $6 \%$, with similar 10 -year cancerfree survival in both groups. ${ }^{67}$ Bach et al ${ }^{68}$ demonstrated that proper selection of patients with $\mathrm{T} 1$ rectal cancer who exhibit favorable tumor biology can undergo TEM and expect similar outcomes as those undergoing radical resection. The authors found that the estimated local recurrence rates for $\mathrm{T} 1$ rectal tumors at 2,3 , and 5 years were $9.5 \%, 12.9 \%$, and
$18.6 \%$, respectively, whereas $\mathrm{T} 2$ tumors exhibited a local recurrence rate starting at $23 \%$ and increased with time, reinforcing that TEM should not be offered to these patients. Moreover, local recurrence-free survival correlated with depth of tumor invasion (T-stage and sm category), tumor diameter, and the presence of intramural lymphovascular Invasion, ${ }^{68}$ making these factors important in the selection of patients for TEM. Heintz et $\mathrm{al}^{35}$ compared the outcomes in patients who underwent TEM versus those who had radical surgery in both "low-risk" and "high-risk" cancers and found that "low-risk" patients had similar 5-year survival as those undergoing radical surgery ( $79 \%$ vs $81 \%$ ) and that patients with "high-risk" tumors were at higher risk of positive lymph nodes after radical surgery (36\%) as well as local recurrence. Stipa et $\mathrm{al}^{69}$ looked at the long-term risk of local and distant recurrence in patients with early stage rectal cancer, including patients with Tis/T1 and T2 disease in 69 patients with a median follow-up of 6.9 years. Although local recurrence rates for Tis/T1 and T2 rectal cancers were similar (median overall recurrence rate of $8.7 \%$ ), the 5 -year disease-specific survival rate was $100 \%$ for both Tis and T1 cancers and $70 \%$ for $\mathrm{T} 2$ rectal cancers, with distant metastasis occurring in $7.2 \%$ of patients who all had $\mathrm{T} 2$ disease, ${ }^{69}$ suggesting TEM should not be used in patients with T2 tumors, unless combined with another modality, such as adjuvant treatment.

There are fewer studies looking at TAMIS and outcomes in early rectal cancer due to its more recent development. Albert et $\mathrm{al}^{70}$ looked at outcomes in their first 50 patients they treated with TAMIS for benign and malignant rectal disease and found an overall recurrence rate of $4.3 \%$ at a mean follow-up of 20 months. For patients with malignant disease, the majority had T1 cancer, with only six patients having T2 or T3 disease, who then underwent immediate radical resection..$^{70}$

\section{Future prospects}

TES for benign and malignant rectal tumors has shown promising results with regard to surgical technique and outcome. However, it can also be used in combination with other treatments such as chemotherapy and radiation to help improve outcomes in more advanced rectal malignancies. One example is the use of postoperative radiotherapy in patients who have undergone TES for suspected benign or early malignancy and were subsequently found to have more advanced malignant disease on pathology. In a healthy, fit patient, the decision should be to perform a formal TME, which is the oncologic standard operation. However, given its high morbidity, certain patients may not be medically fit for 
this radical surgery or refuse to possibly end up with a stoma. These patients can undergo adjuvant radiation therapy to the rectum with the goal of eradicating any residual disease or any possible positive lymph nodes in the mesorectum, resulting in less morbidity and improved functional outcomes. Ramirez et $\mathrm{al}^{71}$ looked at patients with "high-risk" T1 and "low-risk" T2 tumors who received radiotherapy following excision and found higher local recurrence rates when compared to radical surgery. However, survival outcomes were similar with a 5-year cancer-specific survival rate of 93\% in the postoperative radiotherapy group. ${ }^{71}$ Nevertheless, it is important to discuss other alternative treatments with patients since this is not the standard of care and patients must understand the risk of recurrence.

The reverse treatment has also been studied, with patients receiving neoadjuvant radiation with the goal of downstaging the tumor and sterilizing the mesorectum in the case of possible lymph node metastasis, followed by local excision. Once again, this approach is not the standard oncologic operation offered for rectal cancer. It does, however, give the surgeon another possible treatment option to spare patients from the possibility of complications and functional disturbances from radical surgery. Guerrieri et $\mathrm{al}^{72}$ challenged this approach in 137 patients with T1 to T3 rectal cancer with the goal of preserving sphincter function using the TEM platform. They found the overall recurrence rate in the neoadjuvant radiotherapy group to be $4 \%$ versus $33 \%$ patients with disease-free survival (highest in the T0-T1 lesions [100\%], less in the T2 [81\%], and lowest in the T3 group [59\%]), suggesting that although this approach is effective, especially for early tumors, it should only be offered to a select group of patients who will not undergo standard conventional surgery. ${ }^{72}$

The use of neoadjuvant chemoradiotherapy followed by surgery for rectal cancel has revolutionized rectal cancer treatment allowing for tumor downstaging, local control of the disease, and further sphincter-preserving surgery for "high-risk" T2 and T3 locally advanced rectal cancers, leading to better functional outcomes for patients. ${ }^{73}$ In fact, some patients have such a good clinical response to neoadjuvant treatment that the argument of not proceeding to surgery and practicing a "watch and wait" approach has been described and is a hot topic at colorectal meetings worldwide. Somewhere within these two practices is the use of neoadjuvant chemoradiotherapy followed by local excision, sparing patients more radical surgery. In a prospective randomized trial, Lezoche et $\mathrm{al}^{74}$ challenged this treatment plan in patients with early, small T2N0M0, grade 1-2 low-rectal cancer compared to laparoscopic TME in a cohort of 100 patients.
The TEM surgery performed, described as endoluminal locoregional resection, is a full-thickness excision including a portion of the underlying mesorectum. Interestingly, the risk of local recurrence or distant metastasis was similar in both the groups, as was the cancer-related survival rate with a better preference toward endoluminal locoregional resection regarding blood loss, operating time, stoma rate, length of stay, and use of pain medication. ${ }^{74}$ A study by Kim et al ${ }^{75}$ challenged the safety and efficacy of local excision by conventional TAE for T2 and T3 distal rectal cancers following chemoradiotherapy in 26 patients, with half of them having T3N0 disease. Sixty-five percent of patients had a complete pathological response, and 35\% had a partial response, after radical surgery. In the complete responders, there were no recurrences at 24 months follow-up, and there was a lowcomplication rate. Despite this, the authors concluded that even with promising results, choosing patients for local excision should be done cautiously, in a select group of patients. ${ }^{75}$ More recently, the ACOSOG Z6041 trial, a prospective, multicenter, single-arm study, challenged chemoradiotherapy followed by local excision for T2N0 rectal cancer showing a high complete pathologic response rate as well as a high rate of negative margins. However, their reported complication rate was high, suggesting that the efficacy of this approach lies in its long-term oncologic outcome. ${ }^{76}$

The potential for the TES platforms continues to grow as they are starting to be used for more radical operations to help improve mesorectal excision for improved oncologic outcome. Its most recent innovative use has been for TME in early rectal cancer. Although still in its early stages, transanal TME has become a hot topic, with courses being offered in expert centers that have become leaders and proponents of this highly sophisticated technique. The creation of this technique was mainly driven by the difficulties encountered during a TME, during both open and laparoscopic surgery, such as poor visualization and difficulty using instruments deep into the pelvis. This created the need for a "down to up" approach in performing a TME, using a hybrid approach. ${ }^{77}$ In the largest series to date, Lacy et $\mathrm{al}^{77}$ looked at a homogeneous group of patients and suggested that this approach can be particularly advantageous in obese males with bulky advanced tumors, resulting in a very good TME specimen, satisfactory intraoperative outcomes, and comparable short-term morbidity and oncologic outcomes to patients undergoing laparoscopic TME. ${ }^{77}$ Pushing the limits of TES has led to other exceptional uses such as a platform to perform natural orifice transluminal endoscopic surgery. One case report by 
Sylla et $\mathrm{al}^{78}$ describes the use of a rigid TEM platform for a rectosigmoid resection in a swine cadaver using transgastric assistance for colon mobilization.

\section{Conclusion}

TES is a 30 -year-old innovation, which has come into new favor in the minimally invasive surgical era. The creation of new technology has put this technique into the hands of willing laparoscopic surgeons worldwide. The safety and efficacy for its use with benign lesions has been well documented. Malignancies should be approached in a multidisciplinary fashion with careful pathologic, radiologic, and oncologic consideration. New indications and its use for transanal TME are currently being explored with promise.

\section{Disclosure}

The authors report no conflicts of interest in this work.

\section{References}

1. Morino M, Allaix ME. Transanal endoscopic microsurgery: what indications in 2013? Gastroenterol Rep. 2013;1(2):75-84.

2. Saclarides TJ. Transanal endoscopic microsurgery. Clin Colon Rectal Surg. 2015;28(3):165-175.

3. Maya A, Vorenberg A, Oviedo M, da Silva G, Wexner SD, Sands D. Learning curve for transanal endoscopic microsurgery: a single-center experience. Surg Endosc. 2014;28(5):1407-1412.

4. Buess GTR, Gunther M, Hutterer F, Hepp M, Pichlmaier H. Endoscopic operative procedure for the removal of rectal polyps. Coloproctology. 1984 1(84):254-261.

5. Kunitake H, Abbas MA. Transanal endoscopic microsurgery for rectal tumors: a review. Perm J. 2012;16(2):45-50.

6. Maslekar S, Pillinger SH, Sharma A, Taylor A, Monson JR. Cost analysis of transanal endoscopic microsurgery for rectal tumours. Colorectal Dis. 2007;9(3):229-234.

7. Farmer KC, Wale R, Winnett J, Cunningham I, Grossberg P, Polglase A. Transanal endoscopic microsurgery: the first 50 cases. ANZ J Surg. 2002;72(12):854-856.

8. Garcia-Florez LJ, Otero-Diez JL. Local excision by transanal endoscopic surgery. World J Gastroenterol. 2015;21(31):9286-9296.

9. Kahler G. TEO: transanal endoscopic operations. The method of choice for minimally invasive removal of rectal tumors. Available from: http:// shop.stopler.nl/documents/ot/teo-transanal-endoscopic-operations.pdf. Accessed May 12, 2016.

10. Atallah S, Albert M, Larach S. Transanal minimally invasive surgery: a giant leap forward. Surg Endosc. 2010;24(9):2200-2205.

11. Martin-Perez B, Andrade-Ribeiro GD, Hunter L, Atallah S. A systematic review of transanal minimally invasive surgery(TAMIS) from 2010 to 2013. Tech Coloproctol. 2014;18(9):775-788.

12. Barendse RM, Dijkgraaf MG, Rolf UR, et al. Colorectal surgeons' learning curve of transanal endoscopic microsurgery. Surg Endosc. 2013; 27(10):3591-602.

13. Saclarides TJ. TEM/local excision: indications, techniques, outcomes, and the future. J Surg Oncol. 2007;96(8):644-650.

14. Barendse RM, van den Broek FJ, van Schooten J, et al. Endoscopic mucosal resection vs transanal endoscopic microsurgery for the treatment of large rectal adenomas. Colorectal Dis. 2012;14(4):e191-e196.

15. Higaki S, Hashimoto S, Harada K, et al. Long-term follow-up of large flat colorectal tumors resected endoscopically. Endoscopy. 2003;35(10): $845-849$.
16. Iishi H, Tatsuta M, Iseki K, et al. Endoscopic piecemeal resection with submucosal saline injection of large sessile colorectal polyps. Gastrointest Endosc. 2000;51(6):697-700.

17. Saito Y, Sakamoto T, Fukunaga S, Nakajima T, Kiriyama S, Matsuda T. Endoscopic submucosal dissection (ESD) for colorectal tumors. Dige Endosc. 2009;21(Suppl 1):S7-S12.

18. Arezzo A, Passera R, Saito Y, et al. Systematic review and meta-analysis of endoscopic submucosal dissection versus transanal endoscopic microsurgery for large noninvasive rectal lesions. Surg Endosc. 2014;28(2): 427-438.

19. Stamos MJ, Murrell Z. Management of early rectal T1 and T2 cancers. Clin Cancer Res. 2007;13(22 Pt 2):6885s-6889s.

20. Lev-CheloucheD, MargelD, Goldman G, Rabau MJ.Transanal endoscopic microsurgery: experience with 75 rectal neoplasms. Dis Colon Rectum. 2000;43(5):662-667; discussion 7-8.

21. Moore JS, Cataldo PA, Osler T, Hyman NH. Transanal endoscopic microsurgery is more effective than traditional transanal excision for resection of rectal masses. Dis Colon Rectum. 2008;51(7):1026-1030; discussion $30-1$.

22. de Graaf EJ, Burger JW, van Ijsseldijk AL, Tetteroo GW, Dawson I, Hop WC. Transanal endoscopic microsurgery is superior to transanal excision of rectal adenomas. Colorectal Dis. 2011;13(7):762-767.

23. Clancy C, Burke JP,Albert MR, O'Connell PR, Winter DC. Transanal endoscopic microsurgery versus standard transanal excision for the removal of rectal neoplasms: a systematic review and meta-analysis. Dis Colon Rectum. 2015;58(2):254-261.

24. Leslie A, Carey FA, Pratt NR, Steele RJ. The colorectal adenomacarcinoma sequence. Br J Surg. 2002;89(7):845-860.

25. Wise PE. Polyps. In: Beck DE, Roberts Pl, Saclarides TJ, Senagore AJ, Stamos MJ, Nasseri Y, editors. The ASCRS Texbook of Colon and Rectal Surgery. 2nd edition. New York: Springer, 2007;pp:625-641.

26. Casadesus D. Surgical resection of rectal adenoma: a rapid review. World J Gastroenterol. 2009;15(31):3851-3854.

27. Allaix ME, Arezzo A, Cassoni P, Famiglietti F, Morino M. Recurrence after transanal endoscopic microsurgery for large rectal adenomas. Surg Endosc 2012;26(9):2594-2600.

28. Elmessiry MM, Van Koughnett JA, Maya A, et al. Local excision of T1 and T2 rectal cancer: proceed with caution. Colorectal Dis. 2014; 16(9):703-709.

29. Bentrem DJ, Okabe S, Wong WD, et al. T1 adenocarcinoma of the rectum: transanal excision or radical surgery? Ann Surg. 2005;242(4):472477; discussion 7-9.

30. Mellgren A, Sirivongs P, Rothenberger DA, Madoff RD, Garcia-Aguilar J. Is local excision adequate therapy for early rectal cancer? Dis Colon Rectum. 2000;43(8):1064-1071; discussion 71-74.

31. Garcia-Aguilar J, Holt A. Optimal management of small rectal cancers: TAE, TEM, or TME? Surg Oncol Clin North Am. 2010;19(4):743-760.

32. Kikuchi R, Takano M, Takagi K, et al. Management of early invasive colorectal cancer. Risk of recurrence and clinical guidelines. Dis Colon Rectum. 1995;38(12):1286-1295.

33. Blumberg D, Paty PB, Guillem JG, et al. All patients with small intramural rectal cancers are at risk for lymph node metastasis. Dis Colon Rectum. 1999;42(7):881-885.

34. Nascimbeni R, Burgart LJ, Nivatvongs S, Larson DR. Risk of lymph node metastasis in T1 carcinoma of the colon and rectum. Dis Colon Rectum. 2002;45(2):200-206.

35. Heintz A, Morschel M, Junginger T. Comparison of results after transanal endoscopic microsurgery and radical resection for T1 carcinoma of the rectum. Surg Endosc. 1998;12(9):1145-1148.

36. Palma P, Horisberger K, Joos A, Rothenhoefer S, Willeke F, Post S. Local excision of early rectal cancer: is transanal endoscopic microsurgery an alternative to radical surgery? Revista Espanola de Enfermedades Digestivas. 2009;101(3):172-178.

37. D'Ambrosio G, Paganini AM, Guerrieri M, et al. Minimally invasive treatment of rectovaginal fistula. Surg Endosc. 2012;26(2):546-550.

38. Darwood RJ, Borley NR. TEMS: an alternative method for the repair of benign recto-vaginal fistulae. Colorectal Dis 2008;10(6):619-620. 
39. Wilbert DM, Buess G, Bichler KH. Combined endoscopic closure of rectourethral fistula. J Urol. 1996;155(1):256-258.

40. Bochove-Overgaauw DM, Beerlage HP, Bosscha K, Gelderman WA. Transanal endoscopic microsurgery for correction of rectourethral fistulae. J Endourol. 2006;20(12):1087-1090.

41. Kato K, Saito T, Matsuda M, Imai M, Kasai S, Mito M. Successful treatment of a rectal anastomotic stenosis by transanal endoscopic microsurgery (TEM) using the contact Nd:YAG laser. Surg Endosc. 1997;11(5): 485-487.

42. Baatrup G, Svensen R, Ellensen VS. Benign rectal strictures managed with transanal resection - a novel application for transanal endoscopic microsurgery. Colorectal Dis. 2010;12(2):144-146.

43. Chung TP, Hunt SR. Carcinoid and neuroendocrine tumors of the colon and rectum. Clin Colon Rectal Surg. 2006;19(2):45-48.

44. Kinoshita T, Kanehira E, Omura K, Tomori T, Yamada H. Transanal endoscopic microsurgery in the treatment of rectal carcinoid tumor. Surg Endosc. 2007;21(6):970-974.

45. Jones H, Cunningham C. Extending the indications: transanal endoscopic surgery for fistula, stricture, and rare tumors. Sem Colon Rectal Surg. 2015;26:45-48.

46. Cawich SO, Mohammed F, Spence R, Albert M, Naraynsingh V. Colonic foreign body retrieval using a modified TAMIS technique with standard instruments and trocars. Case Reports Emerg Med. 2015;2015:815616.

47. Halefoglu AM, Yildirim S, Avlanmis O, Sakiz D, Baykan A. Endorectal ultrasonography versus phased-array magnetic resonance imaging for preoperative staging of rectal cancer. World J Gastroenterol. 2008;14(22): 3504-3510.

48. Bipat S, Glas AS, Slors FJ, Zwinderman AH, Bossuyt PM, Stoker J. Rectal cancer: local staging and assessment of lymph node involvement with endoluminal US, CT, and MR imaging - a meta-analysis. Radiology. 2004;232(3):773-783.

49. NCCN guidelines: Rectal Cancer; Surveillance. 2016:14. Available from: https://www.nccn.org/professionals/physician_gls/pdf/rectal_blocks.pdf. Accessed May 1, 2016.

50. Kumar AS, Coralic J, Kelleher DC, Sidani S, Kolli K, Smith LE. Complications of transanal endoscopic microsurgery are rare and minor: a single institution's analysis and comparison to existing data. Dis Colon Rectum. 2013;56(3):295-300.

51. Gavagan JA, Whiteford MH, Swanstrom LL. Full-thickness intraperitoneal excision by transanal endoscopic microsurgery does not increase short-term complications. Am J Surg. 2004;187(5):630-634.

52. Molina G, Bordeianou L, Shellito P, Sylla P. Transanal endoscopic resection with peritoneal entry: a word of caution. Surg Endosc. Epub 2015 Aug 13.

53. Guerrieri M, Baldarelli M, Morino M, et al. Transanal endoscopic microsurgery in rectal adenomas: experience of six Italian centres. Dig Liver Dis. 2006;38(3):202-207.

54. Guerrieri M, Baldarelli M, de Sanctis A, Campagnacci R, Rimini M, Lezoche E. Treatment of rectal adenomas by transanal endoscopic microsurgery: 15 years' experience. Surg Endosc. 2010;24(2):445-449.

55. Flexer SM, Durham-Hall AC, Steward MA, Robinson JM. TEMS: results of a specialist centre. Surg Endosc. 2014;28(6):1874-1878.

56. Herman RM, Richter P, Walega P, Popiela T. Anorectal sphincter function and rectal barostat study in patients following transanal endoscopic microsurgery. Int Colorectal Dis. 2001;16(6):370-376.

57. Jin Z, Yin L, Xue L, Lin M, Zheng Q. Anorectal functional results after transanal endoscopic microsurgery in benign and early malignant tumors. World J Surg. 2010;34(5):1128-1132.

58. Zhang HW, Han XD, Wang Y, Zhang P, Jin ZM. Anorectal functional outcome after repeated transanal endoscopic microsurgery. World $J$ Gastroenterol. 2012;18(40):5807-5811.

59. Allaix ME, Rebecchi F, Giaccone C, Mistrangelo M, Morino M. Longterm functional results and quality of life after transanal endoscopic microsurgery. Br J Surg. 2011;98(11):1635-1643.
60. Karakayali FY, Tezcaner T, Moray G. Anorectal function and outcomes after transanal minimally invasive surgery for rectal tumors. J Minimal Access Surg. 2015;11(4):257-262.

61. Verseveld M, Barendse RM, Gosselink MP, Verhoef C, de Graaf EJ, Doornebosch PG. Transanal minimally invasive surgery: impact on quality of life and functional outcome. Surg Endosc. Epub $2015 \mathrm{Jul} 4$.

62. Lee TG, Lee SJ. Transanal single-port microsurgery for rectal tumors: minimal invasive surgery under spinal anesthesia. Surg Endosc. 2014; 28(1):271-280.

63. Buess G, Mentges B, Manncke K, Starlinger M, Becker HD. Technique and results of transanal endoscopic microsurgery in early rectal cancer. Am J Surg. 1992;163(1):63-69; discussion 9-70.

64. Smith LE, Ko ST, Saclarides T, Caushaj P, Orkin BA, Khanduja KS. Transanal endoscopic microsurgery. Initial registry results. Dis Colon Rectum. 1996;39(10 Suppl):S79-S84.

65. Ganai S, Kanumuri P, Rao RS, Alexander AI. Local recurrence after transanal endoscopic microsurgery for rectal polyps and early cancers. Ann Surg Oncol. 2006;13(4):547-556.

66. Said S, Stippel D. Transanal endoscopic microsurgery in large, sessile adenomas of the rectum. A 10-year experience. Surg Endosc. 1995; 9(10):1106-1112.

67. Borschitz T, Heintz A, Junginger T. The influence of histopathologic criteria on the long-term prognosis of locally excised pT1 rectal carcinomas: results of local excision (transanal endoscopic microsurgery) and immediate reoperation. Dis Colon Rectum. 2006;49(10):1492-1506; discussion 500-505.

68. Bach SP, Hill J, Monson JR, et al. A predictive model for local recurrence after transanal endoscopic microsurgery for rectal cancer. Br J Surg. 2009;96(3):280-290.

69. Stipa F, Burza A, Lucandri G, et al. Outcomes for early rectal cancer managed with transanal endoscopic microsurgery: a 5-year follow-up study. Surg Endosc. 2006;20(4):541-545.

70. Albert MR, Atallah SB, deBeche-Adams TC, Izfar S, Larach SW. Transanal minimally invasive surgery (TAMIS) for local excision of benign neoplasms and early-stage rectal cancer: efficacy and outcomes in the first 50 patients. Dis Colon Rectum. 2013;56(3):301-307.

71. Ramirez JM, Aguilella V, Valencia J, et al. Transanal endoscopic microsurgery for rectal cancer. Long-term oncologic results. Int J Colorectal Dis. 2011;26(4):437-443.

72. Guerrieri M, Feliciotti F, Baldarelli M, et al. Sphincter-saving surgery in patients with rectal cancer treated by radiotherapy and transanal endoscopic microsurgery: 10 years' experience. Dig Liver Dis. 2003;35(12): 876-880.

73. Habr-Gama A, Perez RO, Kiss DR, et al. Preoperative chemoradiation therapy for low rectal cancer. Impact on downstaging and sphinctersaving operations. Hepatogastroenterol. 2004;51(60):1703-1707.

74. Lezoche E, Baldarelli M, Lezoche G, Paganini AM, Gesuita R, Guerrieri M. Randomized clinical trial of endoluminal locoregional resection versus laparoscopic total mesorectal excision for T2 rectal cancer after neoadjuvant therapy. Br J Surg. 2012;99(9):1211-1218.

75. Kim CJ,YeatmanTJ,Coppola D, etal.Local excision of 2 andT3 rectal cancers after downstaging chemoradiation. Ann Surg. 2001;234(3):352-358; discussion 8-9.

76. Garcia-Aguilar J, Shi Q, Thomas CR Jr, et al. A phase II trial of neoadjuvant chemoradiation and local excision for $\mathrm{T} 2 \mathrm{~N} 0$ rectal cancer: preliminary results of the ACOSOG Z6041 trial. Ann Surg Oncol. 2012;19(2): 384-91.

77. Lacy AM, Tasende MM, Delgado S, et al. Transanal total mesorectal excision for rectal cancer: outcomes after 140 patients. J Am Coll Surg. 2015;221(2):415-423.

78. Sylla P, Willingham FF, Sohn DK, Gee D, Brugge WR, Rattner DW. NOTES rectosigmoid resection using transanal endoscopic microsurgery (TEM) with transgastric endoscopic assistance: a pilot study in swine. J Gastrointest Surg. 2008;12(10):1717-1723. 
Open Access Surgery is an international, peer-reviewed, open access journal that focuses on all aspects of surgical procedures and interventions. Patient care around the peri-operative period and patient outcomes post surgery are key topics for the journal. All grades of surgery from minor cosmetic interventions to major surgical procedures are covered. Novel techniques and the

Submit your manuscript here: https://www.dovepress.com/open-access-surgery-journal
Dovepress

utilization of new instruments and materials, including implants and prostheses that optimize outcomes constitute major areas of interest. The manuscript management system is completely online and includes a very quick and fair peer-review system, which is all easy to use. Visit http://www.dovepress.com/ testimonials.php to read real quotes from published authors. 